\title{
On Circulant Involutory MDS Matrices
}

\author{
Victor Cauchois* \\ Pierre Loidreau ${ }^{\dagger}$ \\ DGA MI and Université de Rennes 1
}

\begin{abstract}
We give a new algebraic proof of the non-existence of circulant involutory MDS matrices with coefficients in fields of characteristic 2 . In odd characteristics we give parameters for the potential existence. If we relax circulancy to $\theta$-circulancy, then there is no restriction to the existence of $\theta$-circulant involutory MDS matrices even for fields of characteristic 2 . Finally, we relax further the involutory definition and propose a new direct construction of almost involutory $\theta$-circulant MDS matrices. We show that they can be interesting in hardware implementations.
\end{abstract}

\section{Introduction}

MDS matrices are redundant parts of systematic generator matrices of MDS codes. For cryptographic applications they offer the maximal possible diffusion between the input and the output of the associated linear application. For the purpose of reducing implementation costs, research focuses on circulant and recursive matrices. Indeed, the matrix-vector product can be computed with only a linear number of multipliers in the size of the input rather than a square number if no additional structure was required. Moreover if it is used in SPN block-ciphers, the computation of the inverse product is necessary for decryption. In this setting it is thus interesting to consider involutory matrices or almost involutory matrices. All these problems were investigated in a large number of research papers:

- Concerning circulant matrices: Apart from the design of AES [DR02], circulantlike MDS matrix constructions were proposed by taking subsquare matrices of Hankel form, see [Aid86, RS85]. In [RL89], the authors showed that generalized Cauchy matrices are redundant part of MDS codes. More recently, [SKOP15] considered lightweight Hadamard-Cauchy matrices. In [GR14], the authors proved that involutory circulant matrices do not exist. In [LW16], the authors showed that relaxing the definition of circulancy allows to build involutory matrices for some parameters. [LS16] found very low cost involutory matrices by replacing multiplication by field elements with invertible linear applications.

- Concerning recursive matrices: In [Ber13], the author used Gabidulin theory to propose a direct construction of recursive MDS matrices. Recursive MDS

*victouf@hotmail.com

${ }^{\dagger}$ Pierre.Loidreau@m4x.org 
matrix constructions were also considered by [AF14] where they constructed recursive matrices from shortened cyclic MDS codes. Such structures form the linear diffusion layer core of PHOTON family of hash function [GPP11] or LED block cipher [GPPR11]. The construction of involutory matrices with recursive structures was investigated in [CLM16] where the authors published a direct construction of MDS matrices, quasi-involutory and which can be recursively implemented with a derived version of an LFSR denoted SLFSR for Skewed Linear Feedback Shift Register. An SLFSR is a logical structure built from a classical LFSR skewed via the action of a Frobenius automorphism.

From a complexity point of view, verifying that a matrix is MDS is strongly exponential since we have to compute all the minors of the matrix and check that they are non-zero. Therefore, it is important to discriminate parameters for which such matrices could exist. Additionally, we would like to provide direct constructions of such matrices even by relaxing constraints. Thus we would not be limited to small size matrices when designing cryptosystems.

In this paper, after recalling some basics of coding theory we introduce a general algebraic framework to study involutory properties of circulant and $\theta$-circulant matrices. These latter matrices form the counterpart of circulant matrices if one considers the $q$-polynomial ring. In a second part we prove some results on the existence and non-existence of involutory circulant MDS matrices. In a third part we extend the results to the $\theta$-circulant matrices and show that this increases the number of degrees of freedom for the choice of MDS matrices. In a final part by relaxing the involutory property we directly construct MDS $\theta$-circulant matrices which are involutory modulo a permutation.

\section{Our contribution}

We provide a new simple proof that circulant involutory matrices do not exist in fields of characteristic 2 . In odd characteristics, we extend the result by using our algebraic framework. This gives some restrictions on the existence of the matrices. By generalizing circulancy and considering $\theta$-circulant matrices, the aforementioned restriction can be raised and involutory $\theta$-circulant matrices may exist even for impossible parameters in the previous case. This approach does not provide a direct construction. To obtain a direct construction, we relax the condition on the matrix to be involutory, by authorizing the action of the Frobenius automorphism and a permutation of the coordinates. These restriction have little impact on the hardware implementation costs.

\section{An algebraic framework}

\subsection{Notations and definitions}

Let $q$ be the power of a prime $p$. Let $\mathbb{F}_{q}$ be the field with $q$ elements and $\mathbb{F}_{q}[X]$ be the polynomial ring with coefficients in $\mathbb{F}_{q}$. We denote by $\mathcal{M}_{m, n}\left(\mathbb{F}_{q}\right)$ the set of matrices with $m$ rows and $n$ columns with coefficients in $\mathbb{F}_{q}$.

Definition 1. Let $\mathcal{C}$ be $a[n, k, d]$ linear code over $\mathbb{F}_{q}$. Then $\mathcal{C}$ is MDS if its mini- 
mum distance d satisfies the Singleton Bound:

$$
d=n-k+1 .
$$

In cryptography, we are more interested in the so-called MDS matrices defined by:

Definition 2. $\mathbf{M} \in \mathcal{M}_{k, n-k}\left(\mathbb{F}_{q}\right)$ is MDS if and only if it satisfies one of the two following properties:

- All its minors are non zero.

- It is the redundant part of the generator matrix of an MDS code $\mathcal{C}$ under systematic form, i.e. this is the matrix $\mathbf{M}$ where

$$
\mathcal{C}=\langle(\mathbf{I} \mid \mathbf{M})\rangle .
$$

In the design of symmetric encryption schemes we usually need invertible matrices. This implies that we only consider square MDS matrices of order $m$. These matrices are redundant part of generator matrices under systematic form of MDS codes of length $2 m$ and dimension $m$.

Definition 3. A matrix $\mathbf{M} \in \mathcal{M}_{m, m}\left(\mathbb{F}_{q}\right)$ is involutory if it satisfies the following equation:

$$
\mathbf{M}^{2}=\mathbf{I}_{m}
$$

\subsection{Circulant matrices and polynomial rings}

For $g \in \mathbb{F}_{q}[X]$, we denote by $w_{t}(g)$ the weight of the polynomial $g$, corresponding to the number of non-zero coefficients of the polynomial $g$. We denote by $\mathbb{F}_{q, m}[X]$ the set of polynomials of degree less than or equal to $m$.

We introduce the following mapping between monic polynomials and circulant matrices:

Definition 4. Let $h(X)=\left(X^{m}-1\right)+\sum_{i=0}^{m-1} h_{i} X^{i} \in \mathbb{F}_{q}[X]$ be a monic polynomial of degree $m$. The circulant matrix associated with $h$ is the matrix defined by:

$$
\mathbf{C}_{h}=\left(\begin{array}{cccc}
h_{0} & h_{1} & \ldots & h_{m-1} \\
h_{m-1} & h_{0} & \ldots & h_{m-2} \\
\vdots & \ddots & \ddots & \vdots \\
h_{1} & h_{2} & \ldots & h_{0}
\end{array}\right)
$$

Remark 1. This mapping is non-standard in the sense that $h_{0}$ is not the constant term of the polynomial $h$ but the constant term translated by 1 .

The following proposition sets the algebraic framework.

Proposition 1. Let $h(X)=\left(X^{m}-1\right)+\sum_{i=0}^{m-1} h_{i} X^{i} \in \mathbb{F}_{q}[X]$ be a monic polynomial of degree $m$ and $\mathbf{C}_{h}$ be the circulant matrix associated with $h$. Then, $\mathbf{C}_{h}$ is the matrix in the basis $\left\{1, X, \ldots, X^{m-1}\right\}$ of the mapping:

$$
\begin{aligned}
& \phi: \mathbb{F}_{q}[X] /\left(X^{m}-1\right) \rightarrow \mathbb{F}_{q}[X] /\left(X^{m}-1\right) \\
& Q(X) \quad \mapsto \quad Q(X) h(X)
\end{aligned}
$$


Proof. By linearity, we only need to verify that it is true for the elements of the canonical basis of $\mathbb{F}_{q}[X] /\left(X^{m}-1\right)$, associated with the canonical basis $\left(e_{k}\right)_{k \leq m-1}$ of $\mathbb{F}_{q}^{m} \cdot \forall k \in\{0, \ldots, m-1\}$ :

$$
\left\{\begin{array}{l}
X^{k} \cdot h(X)=\sum_{i=0}^{m} X^{k} h_{i} X^{i}=\sum_{i=0}^{m-k-1} h_{i} X^{i+k}+\sum_{i=1}^{k} h_{m-k-1+i} X^{i} \\
e_{k} \cdot \mathbf{C}_{h}=\left(h_{m-k}, \ldots, h_{m-1}, h_{0}, \ldots, h_{m-k-1}\right)
\end{array}\right.
$$

\section{$2.3 \quad \theta$-circulant matrices and $q$-polynomials rings}

Previous framework can be extended to q-polynomial ring: Let $\theta$ be a $\mathbb{F}_{q}$-automorphism of $\mathbb{F}_{q^{m}}$. Consider the set $\left\{\sum_{i} g_{i} X^{i}, g_{i} \in \mathbb{F}_{q^{m}}\right\}$ with the two following operations:

- Addition: usual addition of polynomials

- Multiplication: $X * a=a^{[1]} * X$ where $a^{[i]}=\theta^{i}(a), \forall i \in \mathbb{Z}$

extended by associativity and distributivity. It forms a ring called $q$-polynomial ring we denote by $\mathbb{F}_{q^{m}}[X ; \theta]$. This ring is left and right Euclidean. To distinguish $q$-polynomials from classical polynomials, we use the notation $A\langle X\rangle$ to refer to an element $A \in \mathbb{F}_{q^{m}}[X ; \theta]$.

For $h \in \mathbb{F}_{q^{m}}[X ; \theta]$, we denote by $w_{t}(h)$ the Hamming weight of the $q$-polynomial $h$, which is its number of non-zero coefficients. We denote by $\mathbb{F}_{q^{m}, m}[X ; \theta]$ the set of $q$-polynomials of degree $\leq m$.

Definition 5. Let $h\langle X\rangle=\left(X^{m}-1\right)+\sum_{i=0}^{m-1} h_{i} X^{i} \in \mathbb{F}_{q^{m}}[X ; \theta]$ be a monic $q$ polynomial of degree $m$. The $\theta$-circulant matrix associated with $h$ is the matrix defined by:

$$
\mathbf{C}_{h, \theta}=\left(\begin{array}{cccc}
h_{0} & h_{1} & \ldots & h_{m-1} \\
h_{m-1}^{[1]} & h_{0}^{[1]} & \ldots & h_{m-2}^{[1]} \\
\vdots & \ddots & \ddots & \vdots \\
h_{1}^{[m-1]} & h_{2}^{[m-1]} & \ldots & h_{0}^{[m-1]}
\end{array}\right)
$$

Proposition 2. Let $h\langle X\rangle=\left(X^{m}-1\right)+\sum_{i=0}^{m-1} h_{i} X^{i} \in \mathbb{F}_{q^{m}}[X ; \theta]$ be a monic qpolynomial of degree $m$ and $\mathbf{C}_{h, \theta}$ be the $\theta$-circulant matrix associated with $h$. Then, the matrix $\mathbf{C}_{h, \theta}$ is the matrix in the basis $\left\{1, X, \ldots, X^{m-1}\right\}$ of the application:

$$
\begin{array}{ccc}
\psi: \mathbb{F}_{q^{m}}[X ; \theta] /\left(X^{m}-1\right) & \rightarrow & \mathbb{F}_{q^{m}}[X ; \theta] /\left(X^{m}-1\right) \\
Q\langle X\rangle & \mapsto & Q\langle X\rangle h\langle X\rangle
\end{array}
$$

Proof. By linearity, we only need to verify that it is true for the elements of the canonical basis of $\mathbb{F}_{q^{m}}[X ; \theta] /\left(X^{m}-1\right)$, associated with the canonical basis $\left(e_{k}\right)_{k \leq m-1}$ of $\mathbb{F}_{q}^{m} \cdot \forall k \in\{0, \ldots, m-1\}$ :

$$
\left\{\begin{array}{l}
X^{k} \cdot h\langle X\rangle=\sum_{i=0}^{m} X^{k} h_{i} X^{i}=\sum_{i=0}^{m-k-1} h_{i}^{[k]} X^{i+k}+\sum_{i=1}^{k} h_{m-k-1+i}^{[k]} X^{i} \\
e_{k} \cdot \mathbf{C}_{h, \theta}=\left(h_{m-k}^{[k]}, \ldots, h_{m-1}^{[k]}, h_{0}^{[k]}, \ldots, h_{m-k-1}^{[k]}\right)
\end{array}\right.
$$




\section{Involutory circulant MDS matrices and polyno- mial rings}

Gupta and Ray in [GR14] proved that circulant involutory MDS matrices do not exist in characteristic 2. Based on the framework introduced in section 2.2 we give an alternative simple proof of this result. Then we extend the result to finite fields of any characteristic.

Based on definition 4, we are able to give an algebraic necessary and sufficient condition for such a matrix to be MDS.

Proposition 3. Let $h(X)=\left(X^{m}-1\right)+\sum_{i=0}^{m-1} h_{i} X^{i} \in \mathbb{F}_{q}[X]$. Let $\mathbf{C}_{h}$ be the circulant matrix associated with $h$. Then, $\mathbf{C}_{h}$ is $M D S$ if and only if: $\forall Q_{1} \in \mathbb{F}_{q, m-1}[X]$ we have

$$
w_{t}\left(Q_{1}\right)+w_{t}\left(Q_{1}(X) h(X) \bmod \left(X^{m}-1\right)\right) \geq m+1 .
$$

Proof.

$$
\begin{aligned}
& \mathbf{C}_{h} \text { is MDS } \\
\Leftrightarrow & \left(I_{m} \mid \mathbf{C}_{h}\right) \text { is the generator matrix of an MDS code } \\
\Leftrightarrow & \forall\left(q_{0}, \ldots, q_{m-1}\right) \in \mathbb{F}_{q}^{m}, w_{t}\left(\left(q_{0}, \ldots, q_{m-1}\right) \cdot\left(I_{m} \mid \mathbf{C}_{h}\right)\right) \geq m+1 \\
\Leftrightarrow & \forall\left(q_{0}, \ldots, q_{m-1}\right) \in \mathbb{F}_{q}^{m}, w_{t}\left(q_{0}, \ldots, q_{m-1}\right)+w_{t}\left(\left(q_{0}, \ldots, q_{m-1}\right) \cdot \mathbf{C}_{h}\right) \geq m+1
\end{aligned}
$$

If one considers $Q_{1}(X)=\sum_{i=0}^{m-1} q_{i} X^{i}$, then $w_{t}\left(q_{0}, \ldots, q_{m-1}\right)=w_{t}\left(Q_{1}\right)$. Since from proposition $1, \mathbf{C}_{h}$ corresponds to the multiplication by $h(X)$ in $\mathbb{F}_{q}[X] /\left(X^{m}-1\right)$, we have:

$$
\left.w_{t}\left(\left(q_{0}, \ldots, q_{m-1}\right) \cdot \mathbf{C}_{h}\right)=w_{t}\left(Q_{1}(X) h(X) \bmod \left(X^{m}-1\right)\right)\right),
$$

which proves the proposition.

Example 1. Let $\mathbb{F}_{2^{4}}$ be defined by $X^{4}+X+1$ and $\alpha$ a root of this polynomial. The matrix $\mathbf{C}_{h}$ associated with $h(X)=\left(X^{4}+1\right)+\alpha^{3} X^{3}+\alpha X^{2}+X+1 \in \mathbb{F}_{2^{4}}[X]$ is a circulant MDS matrix.

$$
\mathbf{C}_{h}=\left(\begin{array}{cccc}
1 & 1 & \alpha & \alpha^{3} \\
\alpha^{3} & 1 & 1 & \alpha \\
\alpha & \alpha^{3} & 1 & 1 \\
1 & \alpha & \alpha^{3} & 1
\end{array}\right)
$$

The involutory property for circulant matrices can be written under algebraic form:

Proposition 4. Let $h(X)=\left(X^{m}-1\right)+\sum_{i=0}^{m-1} h_{i} X^{i} \in \mathbb{F}_{q}[X]$. Let $\mathbf{C}_{h}$ be the circulant matrix associated with $h$. Then, $\mathbf{C}_{h}$ is involutory if and only if $h(X)^{2}=$ $1 \bmod \left(X^{m}-1\right)$.

Proof. This proposition comes directly from proposition 1 and from the definition of involutory matrices.

A natural question is to determine whether circulant matrices both MDS and involutory exist. A partial answer in characteristic 2 fields for even size matrices was given in [GR14]. The following theorem simplifies and extends the proofs to even size MDS matrices in finite fields of any characteristic. 
Theorem 1. Let $d \geq 2$. There are no involutory circulant MDS matrix of size $2 d$ over fields of characteristic $p \geq 2$.

Proof. Suppose that $\mathbf{C}_{h}$ is an involutory circulant MDS matrix of size $2 d$ with entries in $\mathbb{F}_{q}$. Let $h \in \mathbb{F}_{q}[X]$ be the polynomial associated with the circulant matrix $\mathbf{C}_{h}$. The proof is separated into two parts depending on the characteristic of the field.

In characteristic 2: We consider the settings of Proposition 1. Let $Q_{1}(X)=X^{d}-1$. By hypothesis $h^{2}(X)-1=(h(X)-1)^{2}=0 \bmod X^{2 d}-1$. Therefore $h(X)-$ $1=0 \bmod X^{d}-1$ therefore $Q_{2}(X)=Q_{1}(X) h(X) \bmod X^{2 d}-1=X^{d}-1$. Hence $w_{t}\left(Q_{1}\right)+w_{t}\left(Q_{2}\right)=4 \leq 2 d+1$ and $\mathbf{C}_{h}$ is not MDS.

In odd characteristics, notice that $X^{2 d}-1=\left(X^{2}-1\right) B(X)$ where $B(X)=\left(X^{2(d-1)}+\right.$ $\left.X^{2(d-2)}+\ldots+1\right)$ has weight $d$. Hence $X^{2 d}-1$ is divisible by $X^{2}-1$ and we have:

$$
\begin{aligned}
\mathbf{C}_{h} \text { involutory } & \Rightarrow h^{2}=1 \bmod \left(X^{2 d}-1\right) \\
& \Rightarrow h^{2}=1 \bmod \left(X^{2}-1\right) \\
& \Rightarrow h^{2}=X^{2} \bmod \left(X^{2}-1\right)
\end{aligned}
$$

Thus, $(h-1)(h+1)=A_{1}(X)\left(X^{2}-1\right)$ and $(h-X)(h+X)=A_{2}(X)\left(X^{2}-1\right)$ where $A_{1}, A_{2} \in \mathbb{F}_{q}[X]$.

We show that $\left(X^{2}-1\right)$ divides necessarily one of the four polynomials $\{h-1, h+$ $1, h-X, h+X\}$ : Suppose $X^{2}-1$ does divides neither $h(X)-1$ nor $h(X)+1$. Since 1 and -1 are the roots of $X^{2}-1$. We have two cases:

1. $h(1)=1$ and $h(-1)=-1$. This implies that $h(X)-X$ contains the roots of $X^{2}-1$ therefore is divisible by $X^{2}-1$;

2. $h(1)=-1$ and $h(-1)=1$. This implies that $h(X)+X$ contains the roots of $X^{2}-1$ therefore is divisible by $X^{2}-1$.

The polynomial $\left(X^{2 d}-1\right)$ divides one of the four polynomials:

$$
\{B(X)(h-1), B(X)(h+1), B(X)(h+X), B(X)(h-X)\}
$$

$\exists A(X) \in \mathbb{F}_{q}[X]$ such that:

- either $B(X) h(X)= \pm B(X)+A(X)\left(X^{2 d}-1\right)$.

- or $B(X) h(X)= \pm X B(X)+A(X)\left(X^{2 d}-1\right)$

Since the degree of $B(X)$ is less than $2 d-2$, the $B(X)$ or $X B(X)$ corresponds to the remainder of $B(X) h(X)$ modulo $X^{2 d}-1$. Moreover since $B(X)$ has weight $d$ so has $X B(X)$ therefore

$$
w_{t}(B(X))+w_{t}(B(X))=w_{t}(B(X))+w_{t}(X B(X))=2 d<2 d+1 .
$$

From proposition 1 this implies that $\mathbf{C}_{h}$ is not MDS, since by taking $Q_{1}(X)=B(X)$ we have $Q_{2}(X)=B(X)$ or $Q_{2}(X)=X B(X)$ and we have found a polynomial that does not satisfy the Hamming weight inequality.

In the particular case of odd characteristics, circulant involutory MDS matrices of odd sizes exist: 
Example 2. Consider $\mathbb{F}_{23}$ be the field with 23 elements. Then, $\mathbf{C}_{h}$, the circulant matrix associated with $h(X)=\left(X^{3}-1\right)+7 X^{2}+7 X+8$ is both involutory and MDS:

$$
C_{g}=\left(\begin{array}{lll}
8 & 7 & 7 \\
7 & 8 & 7 \\
7 & 7 & 8
\end{array}\right)
$$

In characteristic 2 there are no such matrices of odd sizes. The result was first proved in [GR14]. Here we give an another proof, which is an immediate consequence of previous results.

Theorem 2. Let $\mathbb{F}_{q}$ be a finite field of characteristic 2 . Let $m \geq 3$ be an odd integer. There are no involutory circulant MDS matrices of size $m$ with entries in $\mathbb{F}_{q}$.

Proof. Suppose that there exists an involutory circulant MDS matrix of size $m$, say $\mathbf{C}_{h}$ associated to the polynomial $h$. Let $g^{\prime}(X)$ be the derivative of $g(X)=\left(X^{m}-1\right)$ that is:

$$
g^{\prime}(X)=m X^{m-1} .
$$

The polynomial $g^{\prime}(X)$ is prime with $g(X)$. Therefore $g(X)$ has exactly $m$ distinct roots. By hypothesis we have that $h(X)^{2}=1 \bmod X^{m}-1$ with $h$ of degree $m$. Therefore there exist some polynomial $A(X)$ such that:

$$
(h(X)+1)^{2}=A(X)\left(X^{m}-1\right) .
$$

Therefore, the $m$ roots of $X^{m}-1$ are also roots of $(h(X)+1)^{2}$ and since they are all simple roots, they are also roots of $h(X)+1$. Therefore, $h(X)+1=0 \bmod X^{m}-1$. Since $h$ is monic and of degree $m$ necessarily $h(X)=X^{m}-1$. Therefore $C_{h}$ is not MDS since some of its entries are equal to 0.

In characteristic 2, involutory circulant MDS matrices are of size 1 or 2 .

Example 3. Let $\mathbb{F}_{2^{4}}$ be defined by $X^{4}+X+1$ and $\alpha$ a root of this polynomial. The matrix $\mathbf{C}_{h}$ associated with $h(X)=\left(X^{2}+1\right)+\alpha^{4} X+\alpha$ is both involutory and MDS.

$$
\mathbf{C}_{h}=\left(\begin{array}{cc}
\alpha & \alpha^{4} \\
\alpha^{4} & \alpha
\end{array}\right)
$$

Namely, $h(X)^{2}=X^{4}+\alpha^{8} X^{2}+\alpha^{8}=X^{4} \bmod \left(X^{2}+1\right)=X^{2} \bmod \left(X^{2}+1\right)=1$ $\bmod \left(X^{2}+1\right)$

In odd characteristics, the following new theorem shows that there are strong constraints on the size of potential involutory circulant MDS matrices.

Theorem 3. Let $m=2 d+1 \geq 3$. Let $\mathbb{F}_{q}$ be a field of odd characteristic. If there are circulant involutory $M D S$ matrices of size $m$ then there exists $A(X), B(X) \in \mathbb{F}_{q}[X]$ such that

- $A(X)$ has degree exactly d

- $\operatorname{gcd}(A(X), B(X))=1$

- $X^{m}-1=A(X) B(X)$ 
Proof. Suppose there exists an involutory circulant MDS matrix of size $m$, say $\mathbf{C}_{h}$ associated to the polynomial $h$. Suppose $X^{m}-1$ is not decomposable into $X^{m}-1=A(X) B(X)$ where $A(X) \in \mathbb{F}_{q}[X]$ has degree exactly $d$. By hypothesis, we have $h^{2}(X)=1 \bmod X^{m}-1$. Therefore there exists some polynomial $P(X)$ such that

$$
(h(X)-1)(h(X)+1)=P(X)\left(X^{m}-1\right)
$$

In fields of odd characteristics, $(h(X)+1)$ and $(h(X)-1)$ are coprime. Thus, either $\operatorname{gcd}\left(X^{m}-1, h(X)-1\right)$ or $\operatorname{gcd}\left(X^{m}-1, h(X)+1\right)$ is of degree at least $d+2$. There exist then either $B_{1} \in \mathbb{F}_{q}[X]$ or $B_{2} \in \mathbb{F}_{q}[X]$ of degree at most $d-1$ such that $B_{1}(X)(h(X)+1)$ or $B_{2}(X)(h(X)-1)$ is divided by $X^{m}-1$. In such a case $w_{t}\left(B_{i}(X)\right) \leq d$. Since $w_{t}\left(B_{i}(X)\right)+w_{t}\left(B_{i}(X)\right) \leq 2 d<2 d+1$, for $i \in\{1,2\}$ and since $B_{1}(X) h(X)=-B_{1}(X) \bmod \left(X^{m}-1\right)$ or $B_{2}(X) h(X)=B_{2}(X) \bmod \left(X^{m}-1\right), \mathbf{C}_{h}$ is not MDS.

We found circulant involutory MDS matrices by exhaustive search whenever the previous factorization existed. Our conjecture is that for the parameters which are not ruled out by the last three theorems, circulant involutory MDS matrices exist.

Conjecture 1. Let $m=2 d+1 \geq 3$. Let $\mathbb{F}_{q}$ be some field of odd characteristic. If $X^{m}-1$ can be decomposed as $A(X) B(X)$ with $A(X) \in \mathbb{F}_{q}[X]$ of degree $d$ and $\operatorname{gcd}(A(X), B(X))=1$, there exist circulant involutory MDS matrices.

\section{$4 \quad \theta$-circulant matrices and $q$-polynomial rings}

Similarly to classical polynomials, we establish algebraic conditions on $q$-polynomials to characterize those whose associated $\theta$-circulant matrix is MDS. We denote by $\bmod _{*} g$ the operation of computing the remainder of the Euclidean division on the right by $g$ :

$$
c\langle X\rangle \bmod _{*} g=r\langle X\rangle \Leftrightarrow c\langle X\rangle=b\langle X\rangle * g\langle X\rangle+r\langle X\rangle, \operatorname{deg}(r)<\operatorname{deg}(g)
$$

Proposition 5. Let $h\langle X\rangle=\left(X^{m}-1\right)+\sum_{i=0}^{m-1} h_{i} X^{i} \in \mathbb{F}_{q^{m}}[X ; \theta]$. Let $\mathbf{C}_{h, \theta}$ be the $\theta$-circulant matrix associated with $h$, under the form (1). Then, $\mathbf{C}_{h, \theta}$ is MDS if and only if $\forall Q_{1} \in \mathbb{F}_{q^{m}, m-1}[X ; \theta]$, we have:

$$
w_{t}\left(Q_{1}\langle X\rangle\right)+w_{t}\left(Q_{1}\langle X\rangle h\langle X\rangle \bmod { }_{*}\left(X^{m}-1\right)\right) \geq m+1
$$

Proof.

$$
\begin{aligned}
& \mathbf{C}_{h, \theta} \text { is MDS } \\
\Leftrightarrow & \left(I_{m} \mid \mathbf{C}_{h, \theta}\right) \text { is the generator matrix of an MDS code } \\
\Leftrightarrow & \forall\left(q_{0}, \ldots, q_{m-1}\right) \in \mathbb{F}_{q^{m}}^{m}, w_{t}\left(\left(q_{0}, \ldots, q_{m-1}\right) \cdot\left(I_{m} \mid \mathbf{C}_{h, \theta}\right)\right) \geq m+1 \\
\Leftrightarrow & \forall\left(q_{0}, \ldots, q_{m-1}\right) \in \mathbb{F}_{q^{m}}^{m}, w_{t}\left(q_{0}, \ldots, q_{m-1}\right)+w_{t}\left(\left(q_{0}, \ldots, q_{m-1}\right) \cdot \mathbf{C}_{h, \theta}\right) \geq m+1
\end{aligned}
$$

If one considers $Q_{1}\langle X\rangle=\sum_{i=0}^{m-1} q_{i} X^{i}$, then $w_{t}\left(q_{0}, \ldots, q_{m-1}\right)=w_{t}\left(Q_{1}\right)$. From proposition 2, we know that $\mathbf{C}_{h, \theta}$ corresponds to right multiplication by $h\langle X\rangle$ in $\mathbb{F}_{q^{m}}[X ; \theta] /\left(X^{m}-1\right)$, we have then:

$$
\left.w_{t}\left(\left(q_{0}, \ldots, q_{m-1}\right) \cdot \mathbf{C}_{h, \theta}\right)=w_{t}\left(Q_{1}\langle X\rangle h\langle X\rangle \bmod { }_{*}\left(X^{m}-1\right)\right)\right),
$$

which proves the proposition. 
Example 4. Let $\mathbb{F}_{2^{4}}$ be defined by $X^{4}+X+1$ and $\alpha$ a root of this polynomial. Let $\theta$ be the automorphism defined by $a \mapsto a^{2}$. The matrix $\mathbf{C}_{h, \theta}$ associated with $h\langle X\rangle=\left(X^{4}+1\right)+\alpha^{10} X^{3}+\alpha X^{2}+X+1 \in \mathbb{F}_{2^{4}}[X ; \theta]$ is a $\theta$-circulant MDS matrix.

$$
\mathbf{C}_{h, \theta}=\left(\begin{array}{cccc}
1 & 1 & \alpha & \alpha^{10} \\
\alpha^{5} & 1 & 1 & \alpha^{2} \\
\alpha^{4} & \alpha^{10} & 1 & 1 \\
1 & \alpha^{8} & \alpha^{5} & 1
\end{array}\right)
$$

The following proposition gives a simple algebraic necessary and sufficient condition on $q$-polynomials so that their associated $\theta$-circulant matrix is involutory.

Proposition 6. Let $h\langle X\rangle=\left(X^{m}-1\right)+\sum_{i=0}^{m-1} h_{i} X^{i} \in \mathbb{F}_{q^{m}}[X ; \theta]$. Let $\mathbf{C}_{h, \theta}$ be the $\theta$-circulant matrix associated with $h$. Then,

$\mathbf{C}_{h, \theta}$ is a involutory if and only if $h\langle X\rangle * h\langle X\rangle=1 \bmod { }_{*}\left(X^{m}-1\right)$.

Proof. This proposition comes directly from proposition 2 and from the definition of involutory matrices.

Therefore, if one relaxes the condition on the MDS matrix from being circulant to being $\theta$-circulant, this is possible to find involutory matrices with implementation friendly (in a normal basis) properties.

Example 5. Let $\mathbb{F}_{2^{4}}$ be defined by $X^{4}+X+1$ and $\alpha$ a root of this polynomial. Let $\theta$ be the automorphism defined by $a \mapsto a^{2}$. The matrix $\mathbf{C}_{h, \theta}$ associated with $h\langle X\rangle=\left(X^{4}+1\right)+\alpha^{7} X^{3}+\alpha^{14} X^{2}+X+\alpha \in \mathbb{F}_{2^{4}}[X ; \theta]$ is a $\theta$-circulant involutory MDS matrix.

$$
\mathbf{C}_{h, \theta}=\left(\begin{array}{cccc}
\alpha & 1 & \alpha^{14} & \alpha^{7} \\
\alpha^{14} & \alpha^{2} & 1 & \alpha^{13} \\
\alpha^{11} & \alpha^{13} & \alpha^{4} & 1 \\
1 & \alpha^{7} & \alpha^{11} & \alpha^{8}
\end{array}\right)
$$

\section{Direct Construction of $\theta$-circulant almost invo- lutory MDS matrices}

In previous sections, we showed that involutory MDS $\theta$-circulant matrices exist. However this does not give an efficient procedure to find them. The procedure consists in

1. Squaring skew-polynomials of degree $m$ and checking if it can be divided by $X^{m}-1$.

2. If this is the case then testing the nullity of all the minors of the associated matrix.

Alone, the complexity of the second step is exponential since the number of minors is by itself exponential. Namely for a matrix of size $m$, the number of minors is equal to

$$
\sum_{i=1}^{m}\left(\begin{array}{c}
m \\
i
\end{array}\right)^{2} \geq 2^{m}-1
$$


Therefore, even for small sizes enumerating the minors becomes rapidly prohibitive. However, by relaxing the involutory condition, we can build directly MDS matrices from Gabidulin codes which are almost involutory. This works for fields of characteristic 2 and matrices of even length.

The idea consists in considering the skewed matrix product $\mathbf{M} \mathbf{M}^{[1]}$, rather than the usual matrix product, where $\mathbf{M}^{[1]}=\left(m_{i, j}^{[1]}\right)$, provided $\mathbf{M}=\left(m_{i, j}\right)$. Computing $\mathbf{M}^{[1]}$ is done by applying Galois automorphism on matrix coefficients. For hardware implementations, this can be easily done in a normal basis by simple routing.

The second step consists in searching matrices such that $\mathbf{M M}^{[1]}$ is a cyclic permutation and not necessarily the identity. Such a matrix $\mathbf{M}$ is called almost-involutory, and can be efficiently implemented in hardware by adding some routing compared to an usual involutory matrix.

Let $m \geq 2$ be an integer. We consider $\mathbb{F}_{2^{2 m}}$ and the Frobenius automorphism, $\theta(a)=a^{[1]}=a^{2}$. Let $\alpha$ be a normal element in $\mathbb{F}_{2^{2 m}}$ and

$$
\mathbf{G}=\left(\begin{array}{cccc}
\alpha^{[0]} & \alpha^{[1]} & \cdots & \alpha^{[2 m-1]} \\
\alpha^{[1]} & \alpha^{[2]} & \cdots & \alpha^{[0]} \\
\vdots & \vdots & \ddots & \vdots \\
\alpha^{[m-1]} & \alpha^{[m]} & \cdots & \alpha^{[m-2]}
\end{array}\right)
$$

The matrix $\mathbf{G}$ is a generator matrix of a [2m, $m, m+1]$ Gabidulin code, [Gab85]. Let

$$
\mathbf{G}_{1}=\left(\alpha^{[2 j+i]}\right)_{i=0, j=0}^{m-1, m-1}
$$

be the $m \times m$ matrix composed of even columns of $\mathbf{G}$, and let

$$
\mathbf{G}_{2}=\left(\alpha^{[2 j+i+1]}\right)_{i=0, j=0}^{m-1, m-1},
$$

be the $m \times m$ matrix composed of odd columns of $\mathbf{G}$.

Theorem 4. Let $\mathbf{M}=\mathbf{G}_{1}^{-1} \mathbf{G}_{2}$, then

- $\mathbf{M}$ is a $\theta^{2}$-circulant MDS matrix

- $\mathbf{M M}^{[1]}$ is a binary circulant permutation matrix, i.e.

$$
\mathbf{M M}^{[1]}=\left(\begin{array}{cccc}
0 & \cdots & 0 & 1 \\
1 & \cdots & 0 & 0 \\
\vdots & \ddots & \vdots & \vdots \\
0 & \cdots & 1 & 0
\end{array}\right)
$$

Let $\delta_{i, j}$ be the Kronecker symbol that is $\delta_{i, j}=1$ if $i=j$ and 0 otherwise. First we need to prove the following lemma:

Lemma 1. Let $\beta_{0}, \ldots, \beta_{m-1}$, be the first row of $\mathbf{G}_{1}^{-1}$, then
1. $\mathbf{G}_{1}^{-1}=\left(\beta_{j}^{[2 i]}\right)_{i=0, j=0}^{m-1, m-1}$
2. $\sum_{u=0}^{m-1} \beta_{u} \alpha^{[u+2 j]}=\delta_{0, j}$, for all $j=0, \ldots, m-1$
3. $\sum_{u=0}^{m-1} \alpha^{[2 u+i]} \beta_{j}^{[2 u]}=\delta_{i, j}$, for all $i, j=0, \ldots, m-1$ 
Proof. Let $\beta_{0}, \ldots, \beta_{m-1}$, be the first row of $\mathbf{G}_{1}^{-1}$, it satisfies trivially the second item of the lemma. Raising this equation to the power [2], we obtain:

$$
\begin{array}{llll}
\sum_{u=0}^{m-1} \beta_{u}^{[2]} \alpha^{[u+2(j+1)]} & =\delta_{0, j}^{[2]}=\delta_{0, j}, \quad \forall j=0, \ldots, m-1 \\
\sum_{u=0}^{m-1} \beta_{u}^{[2]} \alpha^{[u+2 j]} & =\delta_{0, j-1}^{[2]}=\delta_{1, j}, \quad \forall j=0, \ldots, m-1
\end{array}
$$

where for $j=m-1$, since $\alpha^{[2 m]}=\alpha$, the equality comes from $\alpha^{[u+2(j+1)]}=\alpha^{[u]}$. Therefore $\beta_{0}^{[2]}, \ldots, \beta_{m-1}^{[2]}$ is the second line of $\mathbf{G}_{1}^{-1}$. By induction we prove the first item of the lemma. The last item stems from the obvious relation $\mathbf{G}_{1}^{-1} \mathbf{G}_{1}=\mathbf{I}=$ $\mathbf{G}_{1} \mathbf{G}_{1}^{-1}$.

Now we prove theorem 4

Proof. Let $\mathbf{M}=\left(m_{i, j}\right)=\mathbf{G}_{1}^{-1} \mathbf{G}_{2}$. By construction, $\mathbf{M}$ is the redundant part of some generator matrix under systematic form of a Gabidulin code of length $2 m$ and of dimension $m$ and is then MDS. From previous lemma, the generic term $m_{i, j}$ of M satisfies:

$$
\begin{array}{ll}
m_{i, j}=\sum_{u=0}^{m-1} \beta_{u}^{[2 i]} \alpha^{[u+1+2 j]}, & \text { for all } i, j=0, \ldots, m-1 \\
m_{i, j}^{[2]}=\sum_{u=0}^{m-1} \beta_{u}^{[2(i+1)]} \alpha^{[u+1+2(j+1)]}, & \text { for all } i, j=0, \ldots, m-1
\end{array}
$$

This implies that $m_{i+1, j+1}=m_{i, j}^{[2]}$, therefore $\mathbf{M}$ is a $\theta^{2}$-circulant MDS matrix. It remains to prove the last item of the theorem. The generic $(i, j) t h$ term of $\mathbf{M M}^{[1]}$ is:

$$
\left(\sum_{k=0}^{m-1} \sum_{u, u^{\prime}=0}^{m-1} \beta_{u}^{[2 i]} \alpha^{[u+2 k]} \beta_{u^{\prime}}^{[2(k+1)]} \alpha^{\left[u^{\prime}+2(j+1)\right]}\right)_{i, j}
$$

Note that the only term in the equation depending on $k$ is $\alpha^{[u+1+2 k]} \beta_{u^{\prime}}^{[2(k+1)]}$. Therefore by summing on $k$ and from the third item of lemma, we obtain:

$$
\sum_{k=0}^{m-1} \alpha^{[u+2 k]} \beta_{u^{\prime}}^{[2(k+1)]}=\delta_{u^{\prime}, u+1}
$$

Therefore, equation (3) becomes

$$
\sum_{u=0}^{m-1} \beta_{u}^{[2 i]} \alpha^{[u+2(j+1)]}=\left(\sum_{u=0}^{m-1} \beta_{u} \alpha^{[u+2(j-i+1)]}\right)^{[2 i]}=\delta_{0,(j-i+1)} .
$$

Theorem 4 establishes that the inverse of $\mathbf{M}^{-1}=\mathbf{M}^{[1]} \mathbf{P}$, where $\mathbf{P}$ is a cyclic permutation matrix. From a hardware implementation point of view, permuting bits consists in routing them, so implies no additional cost. If the chosen basis is normal, then the product with $\mathbf{M}^{[1]}$ can be implemented easily with the multipliers implemented for $\mathbf{M}$.

The procedure to construct the matrices is:

1. Choose $\alpha$ a normal element in $\mathbb{F}_{2^{2 m}}$. 

2. Build $\mathbf{G}_{1}=\left(\alpha^{[2 j+i]}\right)_{i=0, j=0}^{m-1, m-1}$ and $\mathbf{G}_{2}=\left(\alpha^{[2 j+i+1]}\right)_{i=0, j=0}^{m-1, m-1}$.
3. Compute $\mathbf{M}=\mathbf{G}_{1}^{-1} \mathbf{G}_{2}$

Example 6. Let $\mathbb{F}_{2^{8}}$ be defined by $X^{8}+X^{4}+X^{3}+X^{2}+1$, and $\alpha$ a root of this polynomial. The element $\alpha^{5}$ is normal and we consider the Gabidulin code over $\mathbb{F}_{2^{8}}$ with generator matrix

$$
\left(\begin{array}{cccccccc}
\alpha^{5} & \alpha^{10} & \alpha^{20} & \alpha^{40} & \alpha^{80} & \alpha^{160} & \alpha^{65} & \alpha^{130} \\
\alpha^{10} & \alpha^{20} & \alpha^{40} & \alpha^{80} & \alpha^{160} & \alpha^{65} & \alpha^{130} & \alpha^{5} \\
\alpha^{20} & \alpha^{40} & \alpha^{80} & \alpha^{160} & \alpha^{65} & \alpha^{130} & \alpha^{5} & \alpha^{10} \\
\alpha^{40} & \alpha^{80} & \alpha^{160} & \alpha^{65} & \alpha^{130} & \alpha^{5} & \alpha^{10} & \alpha^{20}
\end{array}\right)
$$

The extraction of the even columns for $\mathbf{G}_{1}$ and the odd columns for $\mathbf{G}_{2}$ gives

$$
\mathbf{M}=\mathbf{G}_{1}^{-1} \mathbf{G}_{2}=\left(\begin{array}{cccc}
\alpha^{98} & \alpha^{116} & \alpha^{132} & \alpha^{232} \\
\alpha^{163} & \alpha^{137} & \alpha^{209} & \alpha^{18} \\
\alpha^{72} & \alpha^{142} & \alpha^{38} & \alpha^{71} \\
\alpha^{29} & \alpha^{33} & \alpha^{58} & \alpha^{152}
\end{array}\right)
$$

and finally

$$
\mathbf{M M}^{[1]}=\left(\begin{array}{cccc}
0 & 0 & 0 & 1 \\
1 & 0 & 0 & 0 \\
0 & 1 & 0 & 0 \\
0 & 0 & 1 & 0
\end{array}\right)
$$

\section{Conclusion}

Together with a general framework of circulant matrices, we give a new algebraic proof of the non-existence of involutory circulant MDS matrices in fields of characteristic 2 .

Relaxing circulancy by considering $q$-polynomials, we draw general necessary and sufficient conditions for a $q$-polynomial to yield a MDS matrix or to yield an involutory matrix. We have seen that it may allow designers to build circulant layers that are MDS and involutory as in example 5.

Finally, relaxing also the involutory condition, we give a new direct construction of quasi-circulant quasi-involutory MDS matrices from Gabidulin codes.

\section{References}

[AF14] D. Augot and M. Finiasz. Direct construction of recursive MDS diffusion layers using shortened $\mathrm{BCH}$ codes. In Progress in Cryptology, volume 8540, pages 3-17. FSE 2014, 2014.

[Aid86] A. K. Aidinyan. On matrices with nondegenerate square submatrices. In Problems of Information Transmission, volume 22, pages 106-108, 1986.

[Ber13] T. P. Berger. Construction of recursive MDS diffusion layers from Gabidulin codes. In Progress in Cryptology-INDOCRYPT 2013, volume LNCS 8250, pages 274-285. Springer, 2013. 
[CLM16] V. Cauchois, P. Loidreau, and N. Merkiche. Direct construction of quasiinvolutory recursive-like mds matrices from 2-cyclic codes. In IACR Transactions on Symmetric Cryptology, volume 2016 issue 2, pages 8098, 2016.

[DR02] J. Daemen and V. Rijmen. The Design of Rijndael - AES - The Advanced Encryption Standard. Springer-Verlag, 2002.

[Gab85] E. M. Gabidulin. Theory of codes with maximal rank distance. In Problems of Information Transmission, 1985.

[GPP11] J. Guo, T. Peyrin, and A. Poschmann. The PHOTON family of lightweight hash functions. In Advances in Cryptology. CRYPTO 2011, 2011.

[GPPR11] J. Guo, T. Peyrin, A. Poschmann, and M. J. B. Robshaw. The LED block cipher. In CHES 2011, pages 326-341, 2011.

[GR14] K. C. Gupta and I. G. Ray. On constructions of circulant MDS matrices for lightweight cryptography. In ISPEC 2014, pages 564-576, 2014.

[LS16] M. Liu and S. M. Sim. Lightweight MDS generalized circulant matrices. In Fast Software Encryption - 23rd International Conference, FSE 2016, Bochum, Germany, March 20-23, 2016, Revised Selected Papers, pages 101-120, 2016.

[LW16] Y. Li and M. Wang. On the construction of lightweight circulant involutory MDS matrices. In Fast Software Encryption - 23rd International Conference, FSE 2016, Bochum, Germany, March 20-23, 2016, Revised Selected Papers, pages 121-139, 2016.

[RL89] R. M. Roth and A. Lempel. On MDS codes via Cauchy matrices. In IEEE transactions on information theory, volume 35, pages 1314-1319, 1989.

[RS85] R. M. Roth and G. Seroussi. On generator matrices of MDS codes. In IEEE transactions on information theory, volume IT-31, pages 826-830, 1985.

[SKOP15] S. M. Sim, K. Khoo, F. Oggier, and T. Peyrin. Lightweight MDS involution matrices. In FSE 2015, 2015. 